\title{
Strategies Influencing Work- Family Balance among Female Faculty: A Study of Bangladesh
}

\author{
Hasanuzzaman Tushar and Bui Nhat Vuong \\ National Institute of Development Administration, Bangkok, Thailand
}

\begin{abstract}
Traditionally women are expected to do all household works. The increasing working women lead to review this social convention and practice the strategies in balancing work-family lives. Last few decades, it has received much importance in conducting research on developed and developing country. In a less developed country like Bangladesh, very few studies had taken place to investigate the educated women in the higher educational sector. This qualitative study, mainly, is based on the unique experiences of female faculty of the private university in Bangladesh and focuses on the strategies they employ in balancing work-family lives. Nine participants were chosen purposefully with maximum variation and conducted in-depth interview. The authors used a thematic analysis of the interview data presented three themes including: (a) supportive environment at family and workplace, (b) time management and setting up the priorities, and (c) locus of control. The study concludes that the female faculty members emphasize more on families rather than work in balancing their work-family lives; but the behavior is different in case of unmarried women or who stay separated from husband, they like to focus on work. The strategies and overall situation of female faculty members were discussed and recommended further research area.
\end{abstract}

Keywords: Working Women, Gender, Work-Family Balance, Higher Education, Culture. 